\title{
Human causality judgments and response rates on DRL and DRH schedules of reinforcement
}

\author{
PHIL REED \\ University College London, London, England
}

\begin{abstract}
The effect of various relationships between a response (an investment made in the context of a game) and an outcome (a return on the investment) on judgments of the causal effectiveness of the response was examined. In Experiment 1, response rates and causal judgments were higher for a differentialreinforcement-of-high-rate (DRH) schedule relative to a variable-ratio (VR) schedule with the same probability of outcome following a response. Response rates were also higher for a DRH than for a variable-intervalschedule matched for reinforcement rate. In Experiment 2, response rates and causal judgments were lower for a differential-reinforcement-of-low-rate schedule relative to a VR schedule with the same probability of outcome following a response. These results corroborate the view that schedules are a determinant of both response rates and causal judgments, and that few current theories of causal judgment explicitly predict this pattern of results.
\end{abstract}

Human judgments regarding the causal effectiveness of a cue vary as a function of the action-outcome contingency. These variations are similar to those noted in the conditioned responding produced by manipulations in event-outcome contingencies. Such correspondences have formed the basis of a number of theoretical accounts of causality judgment (see Allan, 1993, and Shanks, 1995, for reviews). A factor that might indicate further the extent of the relationship between casual effectiveness and the action-outcome contingency is the impact of schedules of reinforcement on human judgments of causal effectiveness. If human judgments of causal effectiveness follow the same pattern as those of nonhuman conditioned responding, a correspondence between response rate and judgment should be found with schedules of reinforcement.

Paradoxically, such a finding might not support the associative views often put forward by proponents of the idea mentioned above. In instrumental conditioning with free-operant schedules of reinforcement, simple contingency effects do not affect response rates in the manner predicted by simple associative models. Free-operant responding maintained by a schedule of reinforcement is dependent on factors other than probability or rate of reinforcement alone (see Zeiler, 1977, for a review). It is unclear whether the pattern of schedule performance can be reduced to any one factor. This has suggested to some (e.g., Morse $\&$ Kelleher, 1970) that schedules are, in themselves, fundamental determinants of behavior.

These data were first presented during the Experimental Analysis of Behaviour Group Meeting, London, 1998. I thank Lisa A. Osborne for her support. Correspondence should be addressed to P. Reed, Department of Psychology, University College London, Gower Street, London, WC1E 6BT England (e-mail: p.reed@ucl.ac.uk).

-Accepted by previous editorial team of Ralph R. Miller
Whether or not such a view is accepted, there are wellestablished findings in this area. For example, response rates are higher on a variable-ratio (VR) schedule than they are on a variable-interval(VI) schedule. However, initial investigation of the influence of these schedules on judgments of causal effectiveness indicates that the level of causal effectiveness attributed to a response made during a VR schedule is low in comparison with that attributed to a response made during a VI schedule that has the same response-outcome probability (Reed, 1993). This finding is opposite to that which would be predicted if human causality judgments could be accounted for by the same processes that influence response rates. In one study, Reed (1992) examined the effect of differential-reinforcement-of-high-rate (DRH) and differential-reinforcement-of-low-rate (DRL) schedules on human judgments of causal effectiveness. A DRH schedule requires responses to be made in close temporal proximity to one another in order to obtain reinforcement. In contrast, a DRL contingency requires that reinforcement be delivered only if a response is separated from the preceding response by a certain minimum amount of time. Reed (1992) found that ratings of the causal effectiveness of responses were lower for a DRH schedule, and higher for a DRL schedule, than for a VR schedule that had the same probability of an outcome following a response.

In sum, these results suggest that ratings of causal effectiveness are lower when a group of responses are made prior to an outcome, as with a VR or DRH schedule (Ferster \& Skinner, 1957), and are higher when responses are made in temporal isolation prior to an outcome, as with a VI or DRL schedule (cf. Morse, 1966). This relationship is opposite to that between the local context of responding at the time of reinforcement and the response rate, which has been previously noted for schedules of reinforcement (Ferster \& Skinner, 1957). 
However, these results do not necessarily invalidate the suggestion that there is a functional equivalence between animal conditioning and human judgments of causality. There are a number of important differences between the procedure adopted by Reed $(1992,1993)$ and those for which the view mentioned above was formulated. For example, it is not clear how the outcome (i.e., a triangle flash) in the studies conducted by Reed $(1992,1993)$ could be viewed as a reinforcer, such as food, used in studies of free-operant conditioning. A schedule that relates a response to an outcome is not necessarily a reinforcement schedule, since there might be no reinforcement involved.

Given these issues, Reed $(1994,1999)$ extended the investigation of causal judgment to situations that more closely resemble free-operant procedures. In those experiments, subjects made "investment" responses from a hypothetical fund, which were reinforced by the addition of an "investment return" to their fund. With this procedure, the relative rating of causal effectiveness with VI and VR schedules was reversed from that in the earlier studies. Responses made during a VR schedule had higher ratings of causal efficacy than did those made during a VI schedule. This result was obtained despite there being equal probabilities of an outcome following a response for each of the two schedules. However, in these later experiments, the response rates were not consistently higher for the VR in comparison with the VI schedule. The failure to obtain typical response-rate differences in these studies (Reed, 1994, 1999) draws into question the putative parallel between the factors controlling human judgments of causal effectiveness and those controlling performance under free-operant schedules.

The discrepancy in schedule-induced rates of response might be explained by the fact that only one session of training was given to the subjects in the studies mentioned above. Response-rate differences for free-operant schedules do not usually emerge until after a number of sessions (see Ferster \& Skinner, 1957; Mathews, Shimoff, Catania, \& Sagvolden, 1977; Wearden \& Clark, 1988). That the appropriate response-rate differences can be established with multiple exposures to schedules of reinforcement through the present procedure has been demonstrated (Reed, 2001 a, 2001b). In these studies, the response rates for VR and VI schedules were found to differ, as they do in typical studies of free-operant schedules. Moreover, judgments of causal effectiveness under the VR and VI schedules of reinforcement varied in the same way as did the response rates. The present experiment sought to corroborate these findings and extend them to contingencies other than those VR and VI schedules. To this end, subjects were studied under DRL and DRH schedules, previously used with the triangle-flash procedures.

If further evidence of correspondence between response rate and causality judgment can be shown, identification of the factors influencing these results would be important. One such factor implicated in the generation of response-rate differences for various schedules in nonhumans concerns the local rate of responding at the time of the reinforcement. When reinforcement follows a response made in temporal isolation, rates of response are low. In contrast, when reinforcement follows responses made close to one another, rates are high (see Morse, 1966, and Peele, Casey, \& Silberberg, 1984, for discussion).

Unfortunately, with the VR and VI schedules that have typically been used in studying this phenomenon, the local rate of responding at the time of reinforcement has not been directly controlled, but measured post hoc. Moreover, the molar characteristics relating overall response rate to reinforcement rate differ with these two schedules, creating a possible confound in the interpretation of the results. In an attempt to overcome this problem, Reed (2001a) altered a VI schedule so that it reinforced only high local rates of responding. With this manipulation, it was found that rates of response and causal judgments became more similar to those for a VR schedule. Although this result indicates the potential importance of the rate of responding at the time of reinforcement, the modified VI schedule still had molar characteristics that promoted lower rates of responding. In the present experiment, this manipulation was expanded through the use of other schedules that directly manipulated local response rates. Contingencies associated with DRH and DRL schedules are defined in terms of the relationship of response rate to the delivery of reinforcement. Through the use of such schedules, more direct evidence of the role of local rates of responding at the time of reinforcement should be gained.

\section{EXPERIMENT 1}

In the first experiment, the properties of a DRH schedule were used to explore the role of local rates of responding. If schedules that deliver reinforcement following temporally close responses lead to overall high rates of responding, and also to high ratings of causal effectiveness for those responses, both of these measures should be higher for a DRH schedule than for a VR schedule that is yoked to it in terms of the number of responses required for reinforcement. Although the VR schedule tends to reinforce short interresponse times (IRTs), it does not require them for reinforcement to occur, as does the DRH schedule. In Experiment 1, this hypothesis was tested, along with the response rate and ratings obtained with a VI schedule yoked to the DRH schedule in terms of rate of reinforcement.

\section{Method}

Subjects. Ten subjects ( 5 male and 5 female), with an age range of 20-58, were recruited. All of the subjects were volunteers, and none received any payment for their participation.

Apparatus. The experiment was conducted in a room in which the subject sat at a table, on which was placed a BBC computer that controlled the video display screen (screen size $24 \mathrm{~cm}$ wide and $17 \mathrm{~cm}$ high), approximately $50 \mathrm{~cm}$ in front of the subject. The subjects could respond to the instructions given to them on the video screen via the computer keyboard.

Procedure. The subjects were presented with the following instructions: 
You have been given the job of testing the effectiveness of investments made in a number of countries. You must test how well your investments in these countries do and report back to the company that hired you.

Your managing director has given you a sum of $£ 5,000$ to invest as you see fit. After a certain time the board will want a report on how well your investments have gone. They will want to know how effective an investment was in that country."

You can make an investment by pressing the SPACE BAR of the computer. Each press will subtract $£ 100$ from your investment fund. You may, or may not, receive income from your investment. Should you receive income, $£ 1,000$ will be added to your fund.

You are free to make an investment at any time. However, due to the nature of the economies it is to your advantage to invest some of the time and not to invest some of the time.

You will be involved in testing three different economies. The relationship between investment and returns will be constant within a particular country, but may well differ from country to country.

After a period of time, you will be asked to report to the board on your activities. They will want to see a good return on your investment. The board will also want you to give an estimate of how effective an investment was in the country you are investigating.

You will be required to give a rating on a scale of 0 to 100 on the success of your investments. Zero means that investments never made money, and 100 means that investments always produced a returnmade money.

The subjects were exposed to three conditions. For the DRH condition, each subject had to make three responses within $2 \mathrm{sec}$ in order to produce an outcome. If the response requirement was not satisfied before the $2 \mathrm{sec}$ had elapsed, the timer started again, and the subject had to make another three responses in $2 \mathrm{sec}$. The number of responses made before these criteria were met for each successive DRH schedule was recorded, and this number was used for the successive ratio requirements for the VR condition. In a third condition, the subjects responded on a VI schedule yoked to the temporal distribution of outcomes obtained in the DRH condition. All subjects were presented with each schedule four times (i.e., there were a total of 12 trials in all). Each trial lasted 3 min. Each subject experienced one trial with the DRH condition prior to one trial with each of the other two conditions in each of the four sets of exposures. The order of presentation of the VR and VI schedules was quasi-randomized across subjects in the four sets of three trials.

Each schedule type was clearly signaled by the words Country 1 , Country 2 , or Country 3 , which remained visible during that condition. When an outcome was presented, $£ 1,000$ was added to the total displayed on the screen, and the words Successful Investment were displayed below the total. Each subject judged the causal effectiveness of the response (investment) after each trial had finished. At the end of each trial, the subject was asked the following:

How effective on a scale of 0 to 100 was an investment in this country?

\section{Results and Discussion}

The mean rate of response in each condition, during each trial, is shown in the top panel of Figure 1. Inspection of the data shows that response rates for the DRH condi-
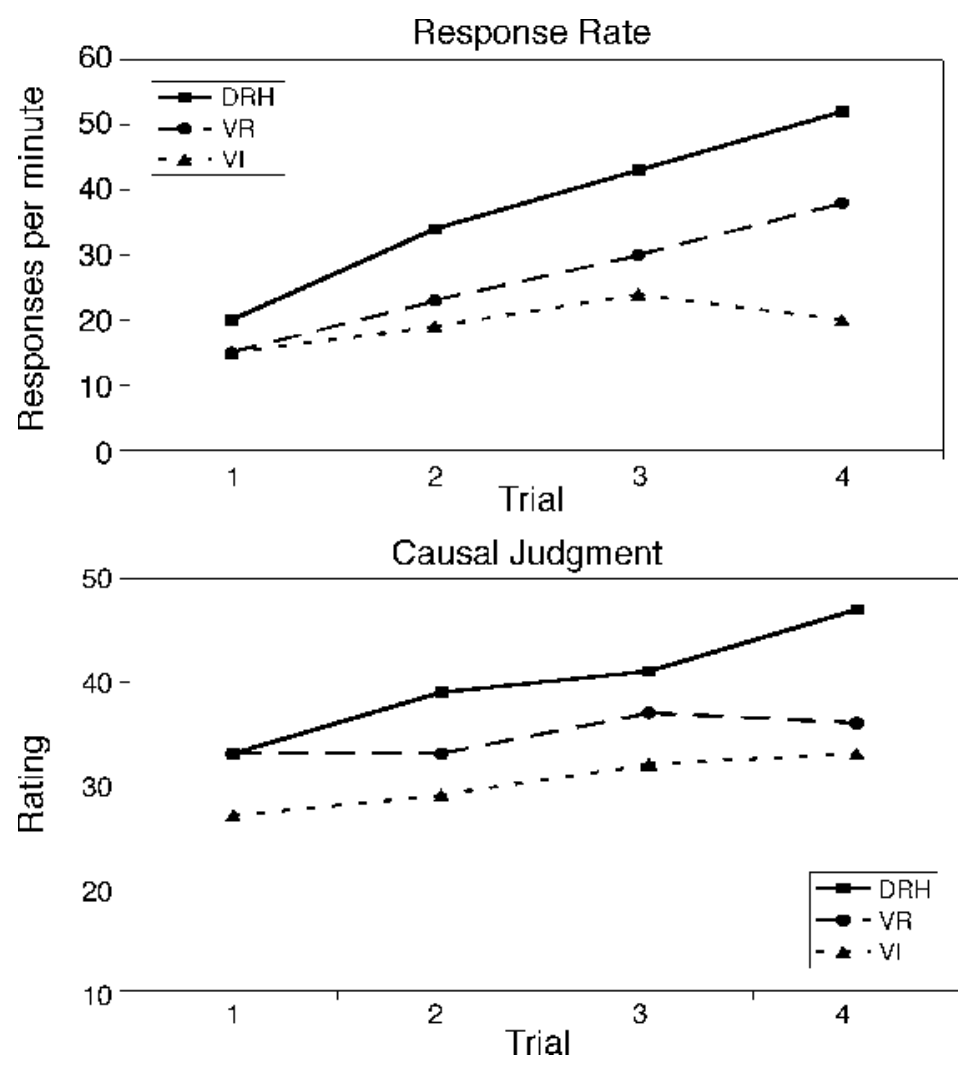

Figure 1. Results from Experiment 1. Top panel: Mean responses per minute over the four exposures to each of the three contingencies. Bottom panel: Mean ratings of the causal effectiveness of responses over the four exposures to each condition. $\mathrm{DRH}=$ differential reinforcement of high rate; $\mathrm{VR}=$ variable ratio; VI = variable interval. 
tion were higher than those for the VR condition. The VR condition, in turn, had higher rates of response than did the VI condition. A two-factor analysis of variance (ANOVA) with schedule (DRH, VR, VI) and trial as factors was conducted on the data. A rejection criterion of $p$ $<.05$ was adopted for this and all subsequent analyses. The ANOVA revealed a significant main effect of schedule $[F(2,18)=8.70]$, a significant main effect of trial $[F(3,27)=26.46]$, and a significant interaction between the two variables $[F(6,54)=7.21]$. The simple main effect of schedule was significant on the last trial $[F(1,54)=$ 4.94]. Tukey's HSD tests conducted on these data revealed a significant difference across all pairwise comparisons of conditions.

Inspection of the ratings of causal effectiveness, displayed in the bottom panel of Figure 1, shows that ratings were typically higher in the DRH condition than in the VR condition. The ratings were also higher in the VR condition than in the VI condition. A two-factor ANOVA (schedule $\times$ trial) revealed a significant main effect of schedule $[F(2,18)=12.26]$, a significant main effect of trial $[F(3,27)=2.83]$, and a significant interaction between the two factors $[F(6,54)=3.99]$. Simple effect analyses revealed a significant effect of schedule for the last trial $[F(2,48)=6.89]$. Tukey's HSD tests conducted on the last trial revealed that the DRH schedule differed significantly from both the VR and the VI schedules.

The mean number of outcomes (and standard deviation) obtained per minute across all four trials was calculated. These data were highly similar for the DRH (4.81 \pm $1.39)$ and VI (4.80 \pm 1.37$)$ schedules and slightly lower for the VR schedule $(3.86 \pm 1.38)$. An ANOVA conducted on these data revealed no significant difference between schedules $(p>.10)$.

The mean probability of an outcome following a response across all four trials for the VR schedule (.04 \pm .01 ) was necessarily the same as that for the DRH schedule $(.04 \pm .01)$, but the probability was higher in the VI schedule (.06 \pm .02$)$. An ANOVA conducted on these data revealed a significant difference between the schedules $[F(2,18)=11.21]$.

The data show that response rates and causality judgments were higher on the DRH schedule than on the VR schedule, despite there being no statistical differences in the rates of reinforcement and probability of reinforcement for the two schedules. The major difference between the schedules was the requirement for several responses to be emitted in close proximity to the reinforcement. Similarly, the DRH schedule produced higher rates of response and ratings of causal effectiveness than did the VI schedule, despite there being no differences in the rate of reinforcement and despite the latter schedule's having a higher probability of an outcome following a response than the DRH schedule. In Experiment 1, previously obtained differences between VR and VI (Reed, 1994, 1999, 2001a, 2001b) were replicated with respect to response rate and causal judgments. Taken together, these data indicate that judgments of causal effectiveness are influenced in a sim- ilar way as response rates under schedules of reinforcement. Both of these measures are high when reinforcement follows a group of responses and when there is a strong relationship between the rate of responding and the rate of reinforcement.

\section{EXPERIMENT 2}

If the schedules that deliver reinforcement for low rates of response lead to overall low rates of responding, and to low ratings of causal effectiveness for those responses, both of these measures should be lower with a DRL than on a VR schedule yoked to it in terms of the number of responses required for reinforcement. Similarly, responding rates and effectiveness judgments should be lower on a DRL schedule than on a VI schedule yoked to it in terms of its reinforcement rate. Although the VI schedule tends to reinforce long IRTs (Ferster \& Skinner, 1957), it does not require them for reinforcement, as does the DRL schedule.

\section{Method}

Subjects and Apparatus. Ten subjects (7 female and 3 male), with an age range of 18-35, volunteered for participation; none were paid. The apparatus was the same as in Experiment 1.

Procedure. Each subject was exposed to three schedules (DRL, VR, and VI) four times during the experiment. One exposure to a DRL schedule was always presented prior to one exposure to each of the other two schedules in each set of four exposures. In the DRL condition, an outcome was presented immediately after a response was made via the space bar. The outcome would occur provided that at least $5 \mathrm{sec}$ had elapsed since the last response or outcome. If a response occurred before $5 \mathrm{sec}$ had elapsed, the DRL time requirement would be reset. The number of responses made before each successive outcome in the DRL schedule was recorded and was played back to the subject so that it could be used for the successive ratio requirements in the subsequent VR schedule. That is, the subjects had to make the same number of responses per outcome in the VR as in the DRL condition. The successive intervals between outcomes in the DRL condition were recorded, and these became the successive interval requirements for the VI condition. In this condition, the first response made after the interval would produce the outcome. Thus, the temporal intervals between outcomes were approximately equal in the DRL and VI conditions. The order of presentation of the VR and VI schedules was quasi-randomized across subjects in the four exposures. All other aspects of the procedure were the same as in Experiment 1.

\section{Results and Discussion}

The mean rate of responding during each exposure to each of the contingencies in Experiment 2 is shown in the top panel of Figure 2. Inspection of the data shows that response rates in the DRL schedule were generally lower than those in both the VR and VI schedules. The VR schedule resulted in a higher rate of responding than did the VI schedule. A two-factor ANOVA (schedule $\times$ trial) revealed a significant main effect of schedule $[F(2,18)=41.03]$, a significant main effect of trial $[F(3,27)=6.46]$, and a significant interaction between the two variables $[F(6,54)=$ 9.35]. The simple main effect of schedule was significant for the last trial $[F(2,50)=8.17]$. Tukey's HSD tests conducted on the last exposure revealed a significant difference across all pairwise comparisons of schedules. 

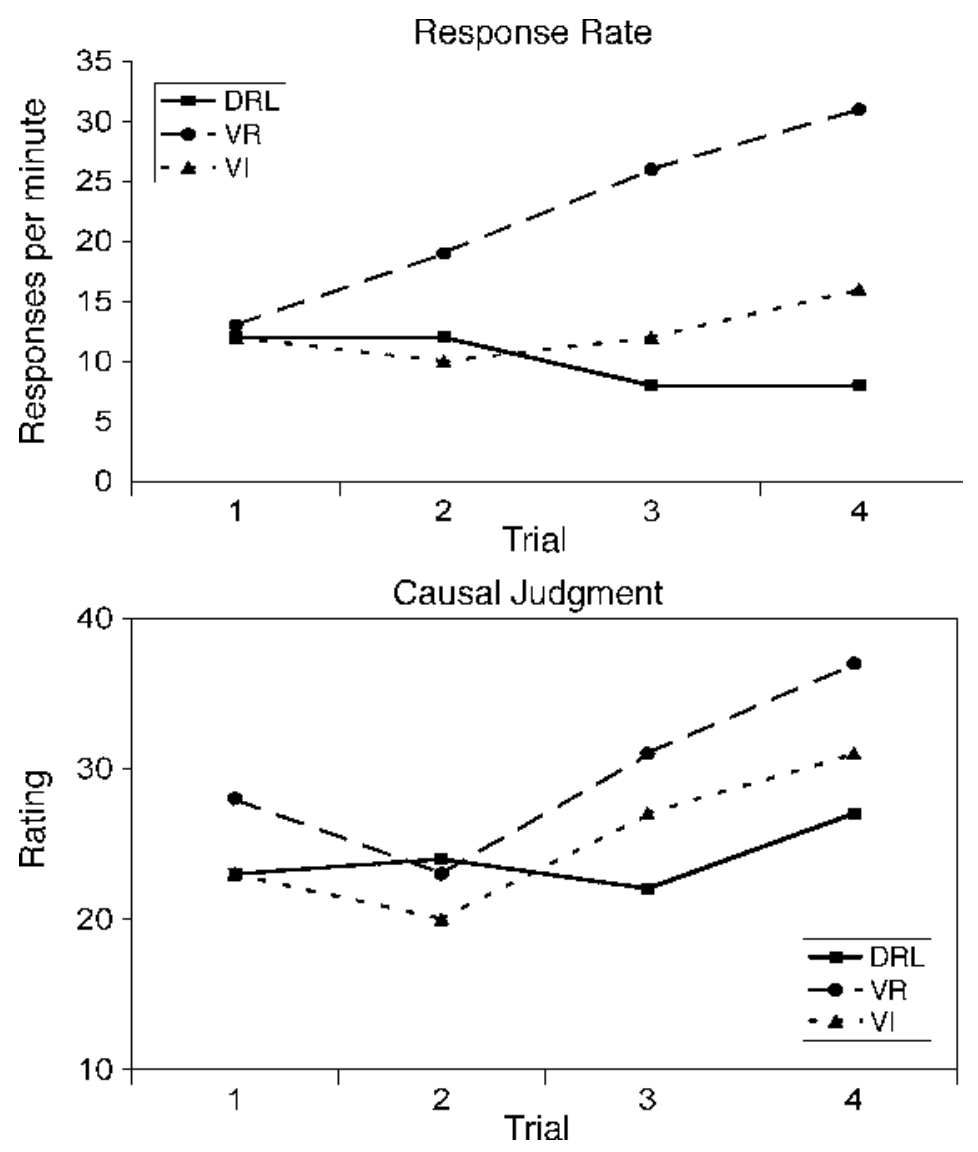

Figure 2. Results from Experiment 2. Top panel: Mean responses per minute over the four exposures to each of the three contingencies. Bottom panel: Mean ratings of the causal effectiveness of responses over the four exposures to each condition. $D R L=$ differential reinforcem ent of low rate; $V R=$ variable ratio; VI = variable interval.

Inspection of the ratings of causal effectiveness, displayed in the bottom panel of Figure 2, shows that the pattern of differences in ratings of causal effectiveness mirrored that for response rates. The ratings were typically higher in the VR condition than in the VI condition. The ratings of causal effectiveness were also higher in the VI condition than in the DRL condition. A two-factor ANOVA (schedule $\times$ exposure) was conducted on these data. This analysis revealed a significant main effect of schedule $[F(2,18)=7.83]$; there was no significant effect of trial, but there was a significant interaction between schedule and exposure $[F(6,54)=6.54]$. Analysis of the simple effect of schedule on the last trial revealed a significant main effect of schedule $[F(2,48)=3.65]$. Tukey's HSD tests revealed a significant difference between all pairwise comparisons of schedules.

The mean number of outcomes obtained per minute across all four trials for the VR condition $(3.80 \pm 2.11)$ was higher than that obtained for both the DRL (1.24 \pm $0.28)$ and VI (1.24 \pm 0.29$)$ conditions. An ANOVA conducted on these data revealed a significant difference between the conditions $[F(2,18)=17.05]$.
The probability of an outcome's following a response across all four trials in the DRL $(.03 \pm .01)$ and VR $(.03 \pm$ $.02)$ conditions was highly similar, but was higher in both these conditions than the VI condition $(.02 \pm .01)$. An ANOVA conducted on these data revealed a significant difference between the conditions $[F(2,18)=9.37]$.

The data show that response rates and causality judgments were lower for the DRL schedule than for the VR schedule. There was no difference in the probability of an outcome's following a response for the two schedules, although there was a difference in the rates of reinforcement for the two schedules. The latter difference could explain the difference in overall response rate, but the ratings of causal effectiveness were different despite equal probabilities of an outcome's following a response for both schedules. The DRL schedule produced lower rates of responding and ratings of causal effectiveness than did the VI schedule. Here, there was no difference in the rate of reinforcement between the schedules, but there was a difference in the probability of the outcome's following a response. The latter measure was lower for the DRL than for the VI schedule. This means that the ratings of causal ef- 
fectiveness could reflect the probability of reinforcement to a response, but not the reinforcement rate. Taken together, these data suggest that neither the probability of an outcome's following a response, nor the rate of reinforcement, is sufficient to accommodate the results in their entirety.

\section{GENERAL DISCUSSION}

In the two present experiments, response rates and judgments of the causal effectiveness of a response appeared to be influenced by similar factors. Both of these measures were high on a schedule that reinforced locally high rates of responding (Experiment 1), and both of these measures were low on a schedule that reinforced locally low rates of responding (Experiment 2). That is, when response rates were high, ratings of the causal effectiveness of a response were also high, and when the response rates were low, ratings of the causal effectiveness of a response were low. Thus, the data suggest that the local rate of responding at the time of reinforcement is a critical determinant of both measures. The data confirm that schedules of reinforcement can exert a strong influence of causal judgments and can extend the range of contingencies for which this is true. Moreover, these data show the importance of local rates of responding in influencing ratings of causal effectiveness, in a way that has not previously been directly studied.

These findings are also important in that no contemporary view of human causality judgment can easily accommodate this pattern of results. The normative, delta-P view (Allan, 1980) suggests that there will be no difference in the ratings of causal effectiveness of responses made in two conditions if the probabilities of outcomes following a response, and in the absence of a response, are equal in both conditions. Similarly, the relative-contiguity view (e.g., Wasserman \& Neunaber, 1986) offers no account of such schedule-induced differences in ratings of causal efficacy. It is possible that the focal delta-P rule (Cheng and Holyoak, 1995; see also Cheng \& Novick, 1992) might go some way toward accommodating the present data. Such a rule suggests that the "window" over which the probability of responses and outcomes is integrated could shift as a function of other factors in the environment. However, a degree of greater specificity would be required regarding when such a modulation of the temporal window of integration would occur before such a rule could adequately accommodate the present data with regard to the influence of the schedule.

One finding that requires discussion concerns the alteration of the effect on humans' rating of causal effectiveness of schedules depending on the procedure adopted (cf. Reed, 1992, 1993, 1994, 1999, 2001a, 2001b). In the present experiment, rates of responding and judgments of causal effectiveness were influenced in the same way by the same factors (see also Reed, 1999, 2001a, 2001b). However, under other circumstances, causal judgments are controlled by the schedule in operation, but are influ- enced in exactly the opposite manner by various schedules of reinforcement, such as when the triangle-flash procedure has been employed (see Reed, 1992, 1993), when the cost of an investment is trivial (Reed, 1994), or when the perceived importance of the investment cost or investment return is low. Under these circumstances, schedules that specify reinforcement following low response rates generate high causal ratings of the response. In contrast, schedules that specify reinforcement for high rates lead to low ratings of causal effectiveness of the response.

There are a number of possible explanations for the different effects on causality judgments that require some brief comment; due to the absence of specific data, these considerations are speculative. It might be that procedures that increase the "importance" of the task (e.g., high returns, high costs, etc.), increase the temporal window over which subjects integrate information. This would allow the molar characteristics of the schedule to dictate performance. Those schedules with a strong response-reinforcement rate relationship would lead to strong perceptions of causal effectiveness (see Dickinson, 1985). A second possibility is that by increasing the importance of the situation, the subjects' attention is focused much more on their responses. If more attention is given to the responses, a group of responses made immediately prior to the reinforcer might create the impression that the responses are connected to the occurrence of outcomes much more readily than if only one response, made in temporal isolation from all others, precedes the outcome.

An alternative explanation for the present results is also worth discussing. The three schedules in Experiment 1 can be ranked according to the degree of control that the subjects could exert over the moment-to-moment changes in reinforcement rate. Subjects exert greater control over moment-to-moment changes in reinforcement rates under DRH schedules than under VR schedules if it is assumed that the DRH schedule is in essence an FR1, in which each set of responses has the possibility of producing a reinforcer. In contrast, under the VR schedule, the minimum number of required responses is greater (i.e., the subject has less control), and is under the control of the schedule. It could be, therefore, that subjects are judging how much control they have over the moment-to-moment changes in reinforcement rate. This could explain the results in Experiment 2, in which the DRL schedule was rated as less effective than the other schedules. Under the DRL schedule, the maximum momentary rate of reinforcement is constrained by the schedule contingency -that is, reinforcement never occurs more frequently than the minimum IRT specified by the schedule. Additionally, with a DRL schedule, responses may also be punished. This could be another reason for subjects to lower their ratings of the momentary effectiveness of responding on a DRL schedule. Although this could explain the present results, it relies on the assumption that the DRH schedule functions like an FR1 schedule. This is something that can occur, but only after extended training (Reed, Schachtman, \& Hall, 1991). It is unclear whether sufficient train- 
ing with the contingencies was given to generate such an effect, although it does remain a possibility. An additional difficulty with this view is that it does not specify what subjects are judging when the costs of a response are low.

Whatever the eventual explanation of the various influences of schedules on causal judgments may be, the present data do demonstrate a similar relationship between the schedule of reinforcement in operation and both the response rate and the judgment of causal effectiveness of responses made under that schedule. This pattern of findings implies that some form of learning-based theory might well be applicable in explaining human judgments of causality, and this explanation could be very similar to that given for nonhuman (and human) response rates. However, it is equally clear that it is difficult for existing views of human judgments of causality to accommodate the present results.

\section{REFERENCES}

Allan, L. G. (1980). A note on measurement of contingency between two binary variables in judgment tasks. Bulletin of the Psychonomic Society, 15, 147-149.

Allan, L. G. (1993). Human contingency judgments: Rule based or associative? Psychological Bulletin, 114, 435-448.

Cheng, P. W., \& Holyoak, K. J. (1995). Complex adaptive systems as intuitive statisticians: Causality, contingency, and prediction. In H. Roitblat \& J.-A. Meyer (Eds.), Comparative approaches to cognitive science (pp. 271-302). Cambridge, MA: MIT Press.

Cheng, P. W., \& Novick, L. R. (1992). Covariation in natural causal induction. Psychological Review, 99, 365-382.

Dickinson, A. (1985). Actions and habits: The development of behavioural autonomy. Philosophical Transactions of the Royal Society of London: Series B, London, 308, 67-78.

Ferster, C. B., \& SKInNer, B. F. (1957). Schedules of reinforcement. New York: Appleton-Century-Croft.

Mathews, B. A., Shimoff, E., Catania, A. C., \& Sagvolden, T. (1977). Unstructured human responding: Sensitivity to ratio and interval contingencies. Journal of the Experimental Analysis of Behavior, 27, 453467 .
Morse, W. H. (1966). Intermittent reinforcement. In W. K. Honig (Ed.), Operant behavior: Areas of research and application (pp. 52-108). Englewood Cliffs, NJ: Prentice-Hall.

Morse, W. H., \& Kelleher, R. T. (1970). Schedules as fundamental determinants of behavior. In W. N. Schoenfeld (Ed.), The theory of reinforcement schedules (pp. 139-185). New York: AppletonCentury-Crofts.

Peele, D. B., Casey, J., \& Silberberg, A. (1984). Primacy of interresponse-time reinforcement in accounting for rates under variableratio and variable-interval schedules. Journal of Experimental Psychology: Animal Behavior Processes, 10, 149-167.

REED, P. (1992). Effect of local context of responding on human judgments of casuality. Memory \& Cognition, 20, 573-579.

REED, P. (1993). Effect of schedule of outcome presentation on human judgments of causality. Quarterly Journal of Experimental Psychology, 46A, 327-345.

REED, P. (1994). Influence of the cost of responding on human causality judgments. Memory \& Cognition, 22, 243-248.

REED, P. (1999). Effect of perceived cost on judgments regarding the efficacy of investment. Journal of Economic Psychology, 20, 657-676.

REED, P. (2001a). Human response rates and causality judgments on schedules of reinforcement. Learning \& Motivation, 32, 332-348.

REED, P. (2001b). Schedules of reinforcement as determinants of human causality judgments and response rates. Journal of Experimental Psychology: Animal Behavior Processes, 27, 187-195.

Reed, P., Schachtman, T. R., \& Hall, G. (1991). Effect of signaled reinforcement on the formation of behavioral units. Journal of Experimental Psychology: Animal Behavior Processes, 17, 475-485.

SHANKS, D. R. (1995). Is human learning rational? Quarterly Journal of Experimental Psychology, 48A, 257-279.

Wasserman, E A., \& Neunaber, D. J. (1986). College students' responding to and rating of contingency relations: The role of temporal contiguity. Journal of the Experimental Analysis of Behavior, 46, 15-35.

WeARden, J. H., \& Clark, R. B. (1988). Interresponse time reinforcement and behavior under aperiodic reinforcement schedules: A case study using computer modelling. Journal of Experimental Psychology: Animal Behavior Processes, 14, 200-211.

ZEILER, M. D. (1977). Schedules of reinforcement: The controlling variables. In W. K. Honig \& J. E. R. Staddon (Eds.), Handbook of operant behavior (pp. 201-232). Englewood Cliffs, NJ: Prentice-Hall.

(Manuscript received March 3, 2002;

revision accepted for publication October 5, 2002.) 